\title{
Colon ischemia in patients with severe COVID-19: a single-center retrospective cohort study of 20 patients
}

\author{
Cui Yang $^{1} \cdot$ Priska Hakenberg $^{1} \cdot$ Christel Wei $^{2} \cdot$ Florian Herrle $^{1} \cdot$ Nuh Rahbari $^{1} \cdot$ Christoph Reißfelder $^{1}$. \\ Julia Hardt ${ }^{1,3}$
}

Accepted: 14 July 2021 / Published online: 29 July 2021

(c) The Author(s), under exclusive licence to Springer-Verlag GmbH Germany, part of Springer Nature 2021

\begin{abstract}
Purpose Thromboembolic complications due to COVID-19 are common in patients requiring critical care and are associated with high morbidity and mortality rates. The aim of this study was to evaluate clinical manifestations and postoperative outcomes after colectomy for COVID-19-associated colonic ischemia in the first versus the second wave of the pandemic. Methods A retrospective cohort study of patients with severe COVID-19 at our institution between 1 March 2020 and 31 January 2021 was performed. All patients with severe COVID-19, requiring critical care and surgical treatment of colonic ischemia, were included. The main outcomes were surgical complications and mortality rate.

Results A total of 190 patients were included of whom 20 (10.5\%) patients underwent surgery for colon ischemia. Preoperatively, D-dimer was elevated, especially in the first wave (median 15.24 (interquartile range 6.00-32.00) vs. 2.09 (1.42-4.28) $\mathrm{mg} / \mathrm{L}$, $p=0.025$ ). Twelve (60\%) patients underwent a (sub)total colectomy, 7 (35\%) a right hemicolectomy, and one patient (5\%) an ileocecal resection. Grade $3 \mathrm{~b}$ complications occurred in one of 5 patients (20\%) in the first and in 9 of 15 patients (60\%) in the second wave. The overall in-hospital mortality was similar in both waves ( $40 \%$ vs. $47 \%$ ), with death occurring after a median stay of 21 days.

Conclusion In this cohort study of patients with severe COVID-19 and colonic ischemia, clinical presentation and laboratory findings varied. However, the majority of patients required (sub)total colectomy. Despite a lower threshold to surgery in the second wave, mortality remained high.
\end{abstract}

Keywords Colon ischemia $\cdot$ COVID-19 disease $\cdot$ SARS-CoV-2 $\cdot$ Colectomy $\cdot$ Mortality

\section{Introduction}

The novel coronavirus disease 2019 (COVID-19) is known to predispose to acute thromboembolic complications, and up to half of the patients with severe thromboembolic events are not salvageable.

Cui Yang and Priska Hakenberg contributed equally to this work.

Julia Hardt

julia.hardt@umm.de

1 Department of Surgery, Medical Faculty Mannheim, Universitätsmedizin Mannheim, Heidelberg University, Mannheim, Germany

2 Department of Medical Statistics, Medical Faculty Mannheim, Heidelberg University, Mannheim, Germany

3 Department of Surgery, Faculty of the University of Heidelberg, University Hospital Mannheim, Theodor-Kutzer-Ufer 1-3, 68167 Mannheim, Germany
After clinicians world-wide observed cases with COVID19-associated thromboembolism, the awareness regarding hypercoagulability has been raised in the course of the pandemic. The management has therefore been optimized to prevent thromboembolic events. However, there is data that almost one-third of critically ill patients with COVID-19 suffer thromboembolic complications despite systemic thromboprophylaxis [1]. If colon ischemia occurs, colectomy is usually inevitable.

The aim of this study was to review clinical courses of patients with COVID-19 and colon ischemia with a special focus on the therapeutic management in the first wave compared to the second wave of the pandemic.

\section{Methods}

We performed a single-center retrospective study (local ethical advisory board ID 2021-802) of all consecutive patients with PCR-confirmed COVID-19 who were mechanically 
ventilated and underwent surgery for colon ischemia between 1 March 2020 and 31 January 2021 (in Germany, the first wave lasted from March 2020 until June 2020 and the second wave from October 2020 until January 2021 [2]).

To identify all eligible patients, we searched our clinical information system for the ICD Codes U07.1, K55.-, K55.0, K55.1, K55.8, and K55.9 and the OPS-Codes 5-453.2 and 5-455.0 to 5-455.y and 5-456 to 5-456.y. Data were managed using REDCap (Research Electronic Data Capture) electronic data capture tools hosted at University Medicine Mannheim.

Quantitative variables are presented by their median values together with the quartiles $\mathrm{Q}_{1}$ and $\mathrm{Q}_{3}$; for qualitative factors, counts and percentages are given. In order to compare two groups, Mann-Whitney $U$ test or Fisher's exact test was performed, as appropriate. The result of a statistical test was considered significant for $p<0.05$. All statistical calculations were done with SAS software, release 9.4 (SAS Institute Inc., Cary, North Carolina, USA).

\section{Results}

We extracted data from 582 consecutive patients with PCRproven COVID-19 (exclusively wild type SARS-CoV-2) between 1 March 2020 and 31 January 2021 of whom 190 required intensive care treatment. Twenty (10.5\%) patients underwent surgery for colon ischemia. In the first wave 5 of 29 patients (17\%) and in the second wave, 15 of 161 patients (9\%) underwent colectomy. Baseline characteristics are summarized in Table 1.

At the time of surgery, 6 patients required extracorporeal membrane oxygenation (ECMO) therapy. Immediately preoperatively, the infection parameters were significantly elevated. D-dimer was also increased, especially in patients of the first wave (15.24 (6.00-32.00) $\mathrm{mg} / \mathrm{L}$ vs. 2.09 (1.42-4.28) $\mathrm{mg} / \mathrm{L}, p=0.025)$. Only a few patients presented with gastrointestinal symptoms. Most patients (16/20) underwent surgery for increasing levels of catecholamines indicating rapid hemodynamic deterioration without further identifiable causes. Computed tomography scan was performed in $95 \%$ of the patients, showing colonic wall thickening (1/19), pneumatosis or portal venous gas (3/19), perforation (1/19), distension of colon (10/19), SMA (superior mesenteric artery), or celiac thrombosis (1/19). Preoperative colonoscopy was performed in 8 patients $(40 \%)$, with no pathological findings in 4 patients. Only one patient had signs of mucosal ischemia in the colonoscopy. The diagnostic parameters are summarized in Table 1.

A (sub)total colectomy was necessary in 12 patients $(60 \%)$, a right hemicolectomy in 7 patients $(35 \%)$ and an ileocecal resection in one patient $(5 \%)$. The pathological examination of the colectomy specimens confirmed the finding of colonic ischemia in all cases. Postoperative surgical complications occurred in 10 patients, and revision surgery was also needed in 10 patients: completion colectomy $(2 / 10)$, small bowel resections for ischemia $(2 / 10)$, cholecystectomy (1/10), operative wound therapy $(5 / 10)$.

Among the whole study cohort, we recorded an in-hospital mortality rate of $45 \%$ in comparison to an overall mortality of $37 \%$ in all COVID-19-positive patients who required critical care (70/190). Deaths occurred after a median of 21 days from admission.

\section{Discussion}

As awareness of the inherent thromboembolism risk in patients with COVID-19 increased during the pandemic, we hypothesized that there may have been fewer thromboembolic complications in the second wave because of improved prevention strategies.

During the studied period, 190 patients were treated for severe COVID-19 at our intensive care units. Of these, 20 patients had to undergo colectomy due to colonic ischemia. Considering the cohort as a whole, some aspects stand out: the median body mass index (BMI) was $31 \mathrm{~kg} / \mathrm{m}^{2}$ corresponding to class 1 obesity, a known risk factor for a complicated course of COVID-19. As a meta-analysis of 12 studies including 405,359 patients revealed, the pooled risk of COVID-19 severity is 1.31 times higher among overweight patients [3]. Another interesting finding was that one-fourth of the patients $(5 / 20,25 \%)$ were under anticoagulant therapy, which raises the question of whether these patients were already at an increased risk of thrombosis before SARS-CoV-2 infection. One could hypothesize that preexisting individual hypercoagulability in addition to COVID-19-induced hypercoagulable state could have increased the risk for thromboembolic events in this subgroup despite prophylactic continuous heparin infusion.

Taking a focused look at the preoperative laboratory values, there are some conspicuous features that are worthy of discussion: first, the D-dimer was relevantly increased, especially in the first wave cohort. Of course, elevated D-dimer levels are common in severe sepsis and disseminated intravascular coagulation due to COVID-19 [4]; nevertheless, the significantly higher values may indicate that patients in the first wave were already in a more progressive septic deterioration and that colonic ischemia was already more advanced when it was diagnosed than in patients in the second wave. Similar trends are seen with respect to procalcitonin, lactate, leucocytes, and C-reactive protein. This could indicate that colonic ischemia was diagnosed earlier in the second wave which led to a faster indication for surgery. In addition, the establishment of a daily ward round by a surgical attending in the medical COVID-19 ICU has certainly contributed to a 
Table 1 Baseline characteristics, diagnostic workup, and postoperative outcomes

\begin{tabular}{|c|c|c|c|c|}
\hline & First wave $(n=5)$ & Second wave $(n=15)$ & $P$ value & Total $(n=20)$ \\
\hline Age (years), median (Q1-Q3) & $68(63-69)$ & $69(61-72)$ & 0.717 & $69(62-72)$ \\
\hline Gender (male/\%) & $2(40 \%)$ & $13(87 \%)$ & 0.073 & $15(75 \%)$ \\
\hline Body mass index $\left(\mathrm{kg} / \mathrm{m}^{2}\right)$ & $32(27.8-33.2)$ & $31.1(28.1-34.6)$ & 0.782 & $31.2(28-33.9)$ \\
\hline $30-35$ & $2(40 \%)$ & $7(47 \%)$ & & $9(45 \%)$ \\
\hline $35-40$ & 0 & $2(13 \%)$ & & $2(10 \%)$ \\
\hline$>40$ & $1(20 \%)$ & $1(7 \%)$ & & $2(10 \%)$ \\
\hline Duration ICU stay (days), median (Q1-Q3) & $27(17-31)$ & $26(17-44)$ & 0.626 & $26.5(17-42)$ \\
\hline \multicolumn{5}{|l|}{ Home medication, $n(\%)$} \\
\hline Home medication, overall & $2(40 \%)$ & $14(93 \%)$ & $\mathbf{0 . 0 3 2}$ & $16(80 \%)$ \\
\hline Anticoagulants & $1(20 \%)$ & $8(53 \%)$ & 0.319 & $9(45 \%)$ \\
\hline Leucocyte count, 10E12/L, median (Q1-Q3) & $24.3(14.4-29.5)$ & $20.3(15.7-24.4)$ & 0.800 & $20.6(15.6-25.0)$ \\
\hline $\mathrm{CRP}, \mathrm{mg} / \mathrm{L}$, median $(\mathrm{Q} 1-\mathrm{Q} 3)$ & $204(164-268)$ & $208(91-274)$ & 1.000 & $206(96.5-271)$ \\
\hline PCT, $\mu \mathrm{g} / \mathrm{L}$, median $(\mathrm{Q} 1-\mathrm{Q} 3)$ & $4.28(4.28-4.28)$ & $1.82(1.18-2.27)$ & 0.546 & $1.87(1.18-4.28)$ \\
\hline D-dimer, mg/L, median (Q1-Q3) & $15.24(6.00-32.00)$ & $2.09(1.42-4.28)$ & 0.025 & $2.18(1.97-6.00)$ \\
\hline Platelet count, 10E9/L, median (Q1-Q3) & $288(211-306)$ & $167(130-280)$ & 0.063 & $187.5(144.5-297)$ \\
\hline PTT, s, median (Q1-Q3) & $68.1(33.7-71.0)$ & $45.5(40.6-62.3)$ & 0.600 & $45.8(39.7-67.0)$ \\
\hline Antithrombin activity, \%, median (Q1-Q3) & $82(75-87)$ & $66(61-80)$ & 0.179 & $75(62-85)$ \\
\hline Lactate, mmol/L, median (Q1-Q3) & $4.2(2.0-6.3)$ & $2.4(1.3-6.5)$ & 0.599 & $3.3(1.4-6.4)$ \\
\hline \multicolumn{5}{|l|}{ Abdominal imaging } \\
\hline Radiography & 0 & $5(33.3 \%)$ & 0.266 & $5(25 \%)$ \\
\hline CT-scan & $5(100 \%)$ & $14(93 \%)$ & 1.000 & $19(95 \%)$ \\
\hline MRI & 0 & 0 & n.c & 0 \\
\hline \multicolumn{5}{|l|}{$\begin{array}{l}\text { Imaging findings, } n(\%) \text {; } \\
\text { Patients with CT-scan }(n=19)\end{array}$} \\
\hline Colonic or rectal wall thickening & 0 & $1(7 \%)$ & 1.000 & $1(5 \%)$ \\
\hline Pneumatosis or PV gas & $1(20 \%)$ & $2(14 \%)$ & 1.000 & $3(16 \%)$ \\
\hline Perforation & $1(20 \%)$ & 0 & 0.263 & $1(5 \%)$ \\
\hline Distension of colon & $1(20 \%)$ & $8(57 \%)$ & 0.303 & $10(53 \%)$ \\
\hline SMA or celiac thrombosis & 0 & $1(7 \%)$ & 1.000 & $1(5 \%)$ \\
\hline Preoperative colonoscopy & $2(40 \%)$ & $6(40 \%)$ & 1.000 & $8(40 \%)$ \\
\hline \multicolumn{5}{|l|}{ Endoscopic findings, $n(\%)$} \\
\hline No pathological findings & $1(50 \%)$ & $3(50 \%)$ & 1.000 & $4(50 \%)$ \\
\hline Mucosal ischemia & 0 & $1(16.6 \%)$ & 1.000 & $1(12.5 \%)$ \\
\hline Transmural ischemia & 0 & 0 & & 0 \\
\hline Bowel dilatation & $1(50 \%)$ & $3(50 \%)$ & 1.000 & $4(50 \%)$ \\
\hline Other & $1(50 \%)$ & $1(16.6 \%)$ & 0.464 & $2(25 \%)$ \\
\hline Norepinephrine level & $5.5(1.1-9.5)$ & $4.7(0.7-6.5)$ & 0.483 & $5.1(0.9-6.5)$ \\
\hline Extent of colon surgery, $n(\%)$ & & & 0.230 & \\
\hline Right hemicolectomy & $2(40 \%)$ & $5(33.3 \%)$ & & $7(35 \%)$ \\
\hline (Sub)total colectomy & $2(40 \%)$ & $10(66.7 \%)$ & & $12(60 \%)$ \\
\hline Ileocecal resection & $1(20 \%)$ & 0 & & $1(5 \%)$ \\
\hline \multicolumn{5}{|l|}{ Revisional surgery, $n(\%)$} \\
\hline Revisional surgery, overall & $1(20 \%)$ & $9(60 \%)$ & 0.303 & $10(50 \%)$ \\
\hline Completion colectomy & 0 & 2 & & 2 \\
\hline Small bowel resection & 1 & 1 & & 2 \\
\hline Cholecystectomy & 0 & 1 & & 1 \\
\hline Other & 0 & 5 & & 5 \\
\hline Surgical complications, $n(\%)$ & $2(40 \%)$ & $7(47 \%)$ & 1.000 & $9(45 \%)$ \\
\hline Further ischemia & 1 & 2 & & 3 \\
\hline Gastrointestinal leakage & 0 & 0 & & 0 \\
\hline
\end{tabular}


Table 1 (continued)

\begin{tabular}{|c|c|c|c|c|}
\hline & First wave $(n=5)$ & Second wave $(n=15)$ & $P$ value & Total $(n=20)$ \\
\hline SSI & 0 & 2 & & 2 \\
\hline Other & 2 & 5 & & 7 \\
\hline Clavien Dindo classification, $n(\%)$ & & & 0.182 & \\
\hline Grade 1 & 0 & 0 & & 0 \\
\hline Grade 2 & $1(20 \%)$ & 0 & & $1(5 \%)$ \\
\hline Grade $3 \mathrm{a}$ & 0 & 0 & & 0 \\
\hline Grade $3 b$ & $1(20 \%)$ & $9(60 \%)$ & & $10(50 \%)$ \\
\hline Grade $4 \mathrm{a}$ & 0 & 0 & & 0 \\
\hline Grade 4b & 0 & 0 & & 0 \\
\hline Grade 5 & 0 & 0 & & 0 \\
\hline Mortality & $2(40 \%)$ & $7(47 \%)$ & 1.000 & $9(45 \%)$ \\
\hline
\end{tabular}

$P$ values in bold type indicate statistical significance. D-dimer conversion factor to SI Unit $\mathrm{nmol} / 1$ is 5.476 . Leucocyte count conversion factor to SI Unit $10^{9}$ is 1000 .

$C R P$ C reactive protein, $C T$ computed tomography, ECMO extracorporeal membrane oxygenation, GI gastrointestinal, $I C U$ intensive care unit, n.c. not calculable, $P C T$ procalcitonin, $P T T$ partial thromboplastin time, $P V$ portal venous, SSI surgical site infection.

faster diagnosis, and the implementation of an extra COVID19 operating room has shortened the interval between the decision to operate and the beginning of the operation at our institution.

That the earliest possible diagnosis of advancing irreversible colonic ischemia is still a challenge becomes evident looking at our results on clinical symptoms and diagnostic workup. Signs of gastrointestinal failure are by far not present in all patients and are sometimes difficult to determine in ventilated patients. Our data is in accordance with a small case series from Spain which found similar gastrointestinal clinical signs in three COVID-19 patients with colon ischemia [5]. This leads to a relatively low threshold to perform diagnostic imaging. However, though $90 \%$ of patients received a CT scan, the findings were often not typical of colonic ischemia. In contrast to this, a review of 22 studies including 31 patients with COVID-19-related gastrointestinal ischemia found that contrast-enhanced abdominal CT scan identified macrovascular thrombosis in almost half of the patients [6]. Similarly, Abdelmohsen reports that in 27 of 40 patients (67.5\%), a CT scan revealed acute vascular pathologies. Therefore, the authors highlight the importance of abdominal CT imaging in COVID-19 patients [7]. Considering the high mortality rate in case of delayed surgery, the therapeutic algorithm for COVID-19 patients should include a rapid indication for surgery, if there are clinical or radiological signs of ischemia.

Comparing the colectomy rates between the first and the second wave, more patients required colectomy in the first wave. This could speak for an optimized management with regard to the prevention of thromboembolic events. However, the pooled colectomy rate among our patient cohort of $10.5 \%(20 / 190)$ is remarkably higher than the reported colectomy rate in ICU patients with admission diagnoses other than COVID-19: a large Finnish retrospective cohort study of 22,936 patients admitted to the ICU showed a colectomy rate of only $0.4 \%$ [8].

Remarkably, right hemicolectomy was necessary in 7 patients $(35 \%)$ and a (sub)total colectomy in 12 patients $(60 \%)$, whereas there was no case of isolated left-sided colonic resection. The reasons for this distribution of ischemia location are unknown. Sotiriadis reports that isolated right colon ischemia accounts for about $10 \%$ of all cases of colon ischemia and has a worse prognosis than nonright-sided colon ischemia; however, it is unclear whether this can be applied to COVID-19 patients [9].

Interestingly, almost one third of the patients with right hemicolectomy had to undergo completion colectomy due to progressive ischemia of the left colon. This leads to the conclusion that in the case of clinical deterioration of patients who had initially undergone only partial colectomy, one must always consider ischemia of the residual colon and should therefore indicate revisional surgery with a low threshold.

The in-hospital mortality rate in our study population was $45 \%$. This is in line with other reports on patients with COVID-19-related acute respiratory distress syndrome [10], and it is also in accordance with the reported in-hospital mortality among patients undergoing colectomy for acute ischemic colitis without an association with COVID-19 [11]. Karagiannidis reports a mortality rate of $53 \%$ of mechanically ventilated patients during the first wave, and this remained unchanged throughout the second wave despite an improvement of the overall management of COVID-19 patients [12]. 
In summary, mortality in COVID-19 patients with colonic ischemia remains high despite improved management and a low threshold to surgery.

\section{Declarations}

Competing interests The authors declare no competing interests.

\section{References}

1. Klok FA, Kruip MJHA, van der Meer NJM et al (2020) Confirmation of the high cumulative incidence of thrombotic complications in critically ill ICU patients with COVID-19: an updated analysis. Thromb Res 191:148-150. https://doi.org/10.1016/j.thromres. 2020.04.041

2. Budweiser S, Bas S, Jörres RA et al (2021) Vergleich der ersten und zweiten Welle hospitalisierter Patienten mit SARS-CoV-2. Dtsch Arztebl Int 118:326-7. https://doi.org/10.3238/arztebl. m2021.0215 (online first)

3. Chowdhury AI, Alam MR, Rabbi MF, Rahman T, Reza S (2021) Does higher body mass index increase COVID-19 severity?: a systematic review and meta-analysis. Obes Med 100340. Published online April 15, 2021. https://doi.org/10.1016/j.obmed.2021. 100340

4. Levi M, Thachil J, Iba T, Levy JH (2020) Coagulation abnormalities and thrombosis in patients with COVID-19. Lancet Haematol 7(6):e438-e440. https://doi.org/10.1016/S2352-3026(20)30145-9

5. Almeida Vargas A, Valentí V, Sánchez Justicia C et al (2020) Severe colon ischemia in patients with severe coronavirus-19
(COVID-19). Rev Esp Enfermedades Dig 112. https://doi.org/ 10.17235/reed.2020.7329/2020

6. Keshavarz P, Rafiee F, Kavandi H, Goudarzi S, Heidari F, Gholamrezanezhad A (2021) Ischemic gastrointestinal complications of COVID-19: a systematic review on imaging presentation. Clin Imaging 73:86-95. https://doi.org/10.1016/j.clinimag.2020.11.054

7. Abdelmohsen MA, Alkandari BM, Abdel Razek AAK, Tobar AM, Gupta VK, Elsebaie N (2021) Abdominal computed tomography angiography and venography in evaluation of hemorrhagic and thrombotic lesions in hospitalized COVID-19 patients. Clin Imaging 79:12-19. https://doi.org/10.1016/j.clinimag.2021.04.002

8. Sipola S, Syrjälä H, Koivukangas V et al (2013) Colectomy in intensive care patients: operative findings and outcomes. World $\mathbf{J}$ Surg 37(2):333-338. https://doi.org/10.1007/s00268-012-1836-1

9. Sotiriadis J, Brandt LJ, Behin DS, Southern WN (2007) Ischemic colitis has a worse prognosis when isolated to the right side of the colon. Am J Gastroenterol 102(10):2247-2252. https://doi.org/10. 1111/j.1572-0241.2007.01341.x

10. Etkin Y, Conway AM, Silpe J et al (2020) Acute arterial thromboembolism in patients with COVID-19 in the New York City area. Ann Vasc Surg. Published online August 28, 2020. https://doi.org/ 10.1016/j.avsg.2020.08.085

11. Reissfelder C, Sweiti H, Antolovic D et al (2011) Ischemic colitis: who will survive? Surgery 149(4):585-592. https://doi.org/10. 1016/j.surg.2010.11.008

12. Karagiannidis C, Windisch W, McAuley DF, Welte T, Busse R (2021) Major differences in ICU admissions during the first and second COVID-19 wave in Germany. Lancet Respir Med. Published online March 5, 2021. https://doi.org/10.1016/S22132600(21)00101-6

Publisher's Note Springer Nature remains neutral with regard to jurisdictional claims in published maps and institutional affiliations. 